\title{
Measuring Crowdedness between Adjacent Stations in an Urban Metro System: a Chinese Case Study
}

\author{
Liudan Jiao $^{1, *}$, Liyin Shen ${ }^{2}$, Chenyang Shuai ${ }^{3}$, Yongtao Tan ${ }^{3}$ and Bei He ${ }^{4}$ \\ 1 School of Economics and Management, Chongqing Jiaotong University, Chongqing 400074, China \\ 2 School of Construction Management and Real Estate, Chongqing University, Chongqing 400044, China; \\ shenliyin@cqu.edu.cn \\ 3 Department of Building \& Real Estate, The Hong Kong Polytechnic University, Hong Kong 999077, China; \\ 18623367874@163.com (C.S.); bstan@polyu.edu.hk (Y.T.) \\ 4 School of Construction Management and Real Estate, Henan University of Economics and Law, \\ Zhengzhou, 450000, China; hebei091@163.com \\ * Correspondence: jld0617@126.com; Tel.: +86-159-2259-6828
}

Received: 21 October 2017; Accepted: 8 December 2017; Published: 13 December 2017

\begin{abstract}
The urban metro system has been widely appreciated as the most important component in urban infrastructures. It plays a critical role in promoting urban social and economic development, and particularly in reducing the urban traffic congestion. However, there are various inherent problems with operating metro systems, which typically involve the crowdedness both at stations and inside vehicles. Both policymakers and academic researchers in China have paid little attention to the crowdedness between metro stations. In order to solve the problem of crowdedness, it is necessary to develop a method to evaluate the level of crowdedness. This work establishes a model to measure the crowdedness between adjacent stations in a metro system based on the load factor principle, passenger standing density, and other factors such as the metro operation schedule and estimations of passenger flows. The Chongqing Metro Line 3 in China is used as a case study to demonstrate the application of the evaluation model. The case study reveals that the model introduced in this study can assist with assessing the crowdedness level between adjacent stations in a metro line. The model is an effective tool for helping the metro management and administration understand the level of crowdedness, apply proper methods to mitigate the crowdedness, and thus improve the quality of the service for those utilizing the metro system.
\end{abstract}

Keywords: urban metro stations; crowdedness; evaluation model; China

\section{Introduction}

The urban metro system has been widely appreciated as the most important component in urban infrastructures [1-4]. It plays an essential role in promoting economic and social development in urban areas [5-7]. Many countries have developed metro systems since the first metro line was built in 1863 in United Kingdom. According to the statistics, 195 cities across the world have developed metro systems between 1863 and 2014 [8]. The number of passengers using the metro systems has been increasing dramatically. For example, the total length of the Beijing metro system reached $604 \mathrm{~km}$ by the end of 2014, including 18 lines; and had achieved a daily ridership of 9.53 million [9]. In contrast, there were only two metro lines in Beijing in 2000 with a total length of $54 \mathrm{~km}$, and the daily ridership was about 1.2 million. The metro system in Beijing is one of the busiest metro systems in the world.

As the number of passengers choosing metro systems for daily transportation increases, crowdedness becomes common in many metro lines, which affects the quality of service. Crowdedness happens when the daily ridership exceeds the number of people for which it was designed. There are many cities worldwide with a total metro length greater than $300 \mathrm{~km}$, such as Beijing, Shanghai, 
London, Moscow, Tokyo, New York, and others. These cities have developed very mature metro systems, but at the same time, they encounter the problem of serious crowdedness in their operating metro systems. In the Chinese cities, reports indicate that ridership per metro line where the metro systems are well developed is higher than those where it is underdeveloped. People often have to wait for the arrival of metro vehicles by joining a long queue, with very crowded passengers both on the platform and inside metro vehicles. This crowdedness situation also becomes more serious during peak hours.

When crowdedness becomes serious, it can lead to many problems, such as those of safety and security, fire accidents, etc. Shen et al. [10] pointed out that "crowdedness" in operating metro systems should be given more attention than utility efficiency when a city is in a more developed stage. Jiang et al. [11] pointed out that the crowded metro system will reduce the comfort of passengers and increase the risks of safety and fire. The study by Tirachini et al. [12] shows that passengers in crowded public transport systems such as metro systems will suffer from travelling. Cox et al. [13] found that crowdedness in a metro system presents a threat both to the development of the rail industry and the health of passengers. Kim et al. [14] suggested that the crowdedness of a metro train is an important measure of passenger satisfaction, which provides a criterion for determining service frequency and the number of cars needed for a metro line. Therefore, it is important to measure the crowdedness of an operating metro system. A proper measurement of a metro system's crowdedness can help management properly understand problems [15], identify the reasons contributing to crowdedness, and formulate a proper management method to solve the problems.

Other research studies have investigated the crowdedness problem in metro systems [16-18]. For example, Kim et al. [17] investigated how the problem of crowdedness affects the passengers' choice for transportation, and found that the problem reduces the overall satisfaction of metro passengers. Some other researchers focus on the crowdedness problem from the perspective of other forms of public transportation, such as rail systems [19-23]. For example, the research findings by Currie [20] demonstrated that the Australia Rail Company successfully managed to solve the problem of rail crowdedness during peak times through adopting a system of different ticket prices for application during different time periods. Wardman and Whelan [21] suggested using the number of standing passengers per square meter as a measurement of the discomfort of passengers in a rail system.

Previous scholars have introduced other indicators to measure the crowdedness of a whole metro system or other public transportation systems. For example, Wu and Ma [24] proposed four key indicators to quantify the crowdedness of a platform in a metro system, including per capita space, per capita longitudinal distance, boarding coefficient, and evacuation coefficient. Chow appreciated [25] that for public access places such as shopping malls and transport interchanges, the waiting time is observed to be the one important component for evacuation under crowded conditions. Tirachini et al. [26] used four main variables-in-vehicle time, the occupancy level of passengers, the number of passengers sitting, and the number of passengers standing-to assess the crowding discomfort in public transport vehicles. Through a comprehensive literature study, it is found that among the various indicators, two quantitative indicators-namely, load factor and passenger standing density (pass $/ \mathrm{m}^{2}$ ) — have been widely adopted to measure the crowding levels of public transportation $[12,21,27-29]$.

For other aspects, metro stations are the key nodes of the metro network system [30]. Different types of metro stations have different functions, as reflected in their regional characteristics, traffic function, and land use [31]. Zhao et al. [32] also pointed out that the ridership of different metro stations in a metro line is different, and identified five key factors affecting the ridership, including employment, road length, feeder bus lines, bicycle park-and-ride (P\&R) spaces, and the transfer dummy variable. Furthermore, the influence of a metro transit network on the urban spatial structure depends primarily on the location of the metro stations [33]. So, whether the distribution of metro stations in a metro line is reasonable or not directly relates to the efficiency and social and economic benefits of metro 
systems [34]. Therefore, as mentioned above, it is imperative to consider the crowdedness between the stations in a metro system from the perspective of the reasonable distribution of the metro stations.

However, both policymakers and academic researchers in China have paid little attention to the crowdedness between metro stations. This papers attempts to fill this gap. The aim of this paper is twofold. First, based on the load factor principle, passenger standing density, and other factors, such as the metro operation schedule and measurements of passenger flow, we present a model to measure the crowdedness between adjacent stations in a metro system (Section 2). Second, the crowdedness of adjacent metro stations of Chongqing Metro Line 3 in China is examined, and relevant management methods and policies for reducing the crowdedness are also discussed (Section 3).

\section{Model Development}

The research starts with understanding the existing methods for evaluating the performance of metro systems. A literature review also helps identify the key variables for assessing the level of crowdedness in an operating metro system. Following the literature review, a quantitative measurement model of crowdedness is developed. The development of this model used relevant design and operation standards for metro system as references.

\subsection{Development of Measurement Model of Crowdedness}

A crowd is commonly defined as a large group of people that are gathered or considered together [35]. Crowd counting is a technique used to count or estimate the number of people in a crowd [36]. The excessive number of passengers using a metro system will generate the crowdedness phenomenon. The study by Jiao et al. [37] suggested measuring the crowdedness of a metro system by considering the difference between the estimated number of daily riders and the actual number, demonstrated as follows:

$$
\theta=\frac{R_{p}}{R_{d}}
$$

where $\theta$ is the level of crowdedness in an operating a metro system; $R_{d}$ is the designed value of daily ridership per kilometer for the metro system, and $R_{p}$ is the actual daily ridership per kilometer of the system.

However, the level of crowdedness at different stations in a metro line is different. A metro line consists of a number of stations. One metro station and its nearest station are considered as two adjacent stations. In order to measure the crowdedness between adjacent stations, model (1) is considered the basis in this study.

In fact, the crowdedness between different adjacent stations is different, as passengers' arrival and departure behavior is different in different stations. Therefore, the measurement model (1) should be modified to measure the level of crowdedness between adjacent stations, which is proposed as follows:

$$
\theta_{s-s}=\frac{R p_{(s-s)}}{R d_{(s-s)}}
$$

where $\theta_{s-s}$ denotes the level of crowdedness between adjacent stations in a metro line; $R d_{(s-s)}$ is the designed value of daily ridership per kilometer per metro line; and $R p_{(s-s)}$ is the actual daily ridership per kilometer between stations.

The value of $R d_{(s-s)}$

In model (2), the value of the parameter $R d_{(s-s)}$ is determined at the design stage of a metro system. It is appreciated that the value of $R d_{(s-s)}$ will be different when different design standards are adopted. $R d_{(s-s)}$ in this study is considered from two main aspects: the designed carrying capacity (or the transportation volume) of the metro vehicle, and the metro operation schedule. According to Li and Hensher [38], many United States (US) transit authorities adopted the load factor (passengers/seat) to evaluate the in-vehicle crowdedness of the rail system. In line with this, this study chooses the 
load factor to represent the designed carrying capacity of a metro vehicle. Furthermore, the designed carrying capacity includes the designed number of seats and the designed stand numbers for a carriage.

Another issue for considering $R d_{(s-s)}$ is the metro operation schedule. The level of crowdedness between adjacent stations will be different during the opening hours of the metro system when different schedules of operation are adopted. In this study, the daily metro operation schedule is used for analysis. Accordingly, the parameter $R d_{(s-s)}$ is defined from the following, model (3):

$$
R_{d(s-s)}=\frac{(x+y) z m}{L_{(s-s)}}
$$

where $L_{(s-s)}$ denotes the length between two adjacent stations; $x$ and $y$ stand for the designed seat numbers and the designed stand numbers in a metro carriage, respectively; $z$ is the number of the carriages in a metro line; and $\mathrm{m}$ is the number of travels of the metro line according to the daily schedule.

The value of $R p_{(s-s)}$

The value of $R p_{(s-s)}$ is obtained as follows:

$$
R_{p(s-s)}=\frac{r_{P(s-s)}}{L_{(s-s)}}
$$

where $r_{p(s-s)}$ stands for the total number of passengers flowing between two adjacent stations per day, and $L_{(s-s)}$ denotes the length between the two adjacent stations.

The values of variables $L_{(s-s)}, x, y, z$, and $m$ in model (3) and (4) can be obtained from the relevant design documents. However, the value of the variable $r_{p(s-s)}$ is the actual passenger flows between two adjacent stations. Passengers' arrival and departure behavior will directly affect the value of $r_{p(s-s)}$. It is considered that the alighting and boarding flow can reflect the passengers' arrival and departure behavior. Figure 1 presents an analytical framework for the passengers' flows of alighting and boarding between stations.

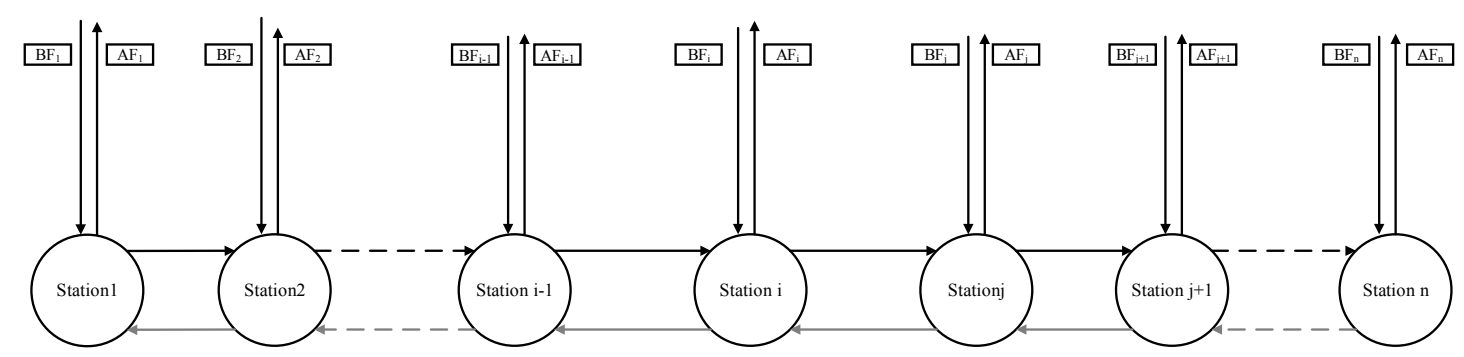

Figure 1. Passenger flows between stations.

In Figure 1, it is assumed that there are $\mathrm{n}$ stations in a metro line. Each individual station has two passenger flows: alighting flow (AF) and boarding flow (BF). $\mathrm{BF}_{i}$ is the value of boarding flow for Station $i$, and $\mathrm{AF}_{i}$ is the value of alighting flow for Station $i$.

In referring to Figure 1 , the boarding passengers, denoted as $\mathrm{BF}_{i}$, can go to other $(n-1)$ stations in the metro line. Accordingly, $(n-1)$ boarding flows will be generated, including $\mathrm{BF}_{i-1}, \mathrm{BF}_{i-2}, \ldots, \mathrm{BF}_{i-(j-1)}$, $\mathrm{BF}_{i-j}, \mathrm{BF}_{i-(j+1)}, \ldots, \mathrm{BF}_{i-n}$, as shown in Figure 2, where $\mathrm{BF}_{i-j}$ is the number of the passengers flowing from Station $i$ to Station ${ }_{j}$. Similarly, the boarding flow for other stations can be established. As a result, the boarding flows for all of the individual stations can be established, as presented in Figure 2. 


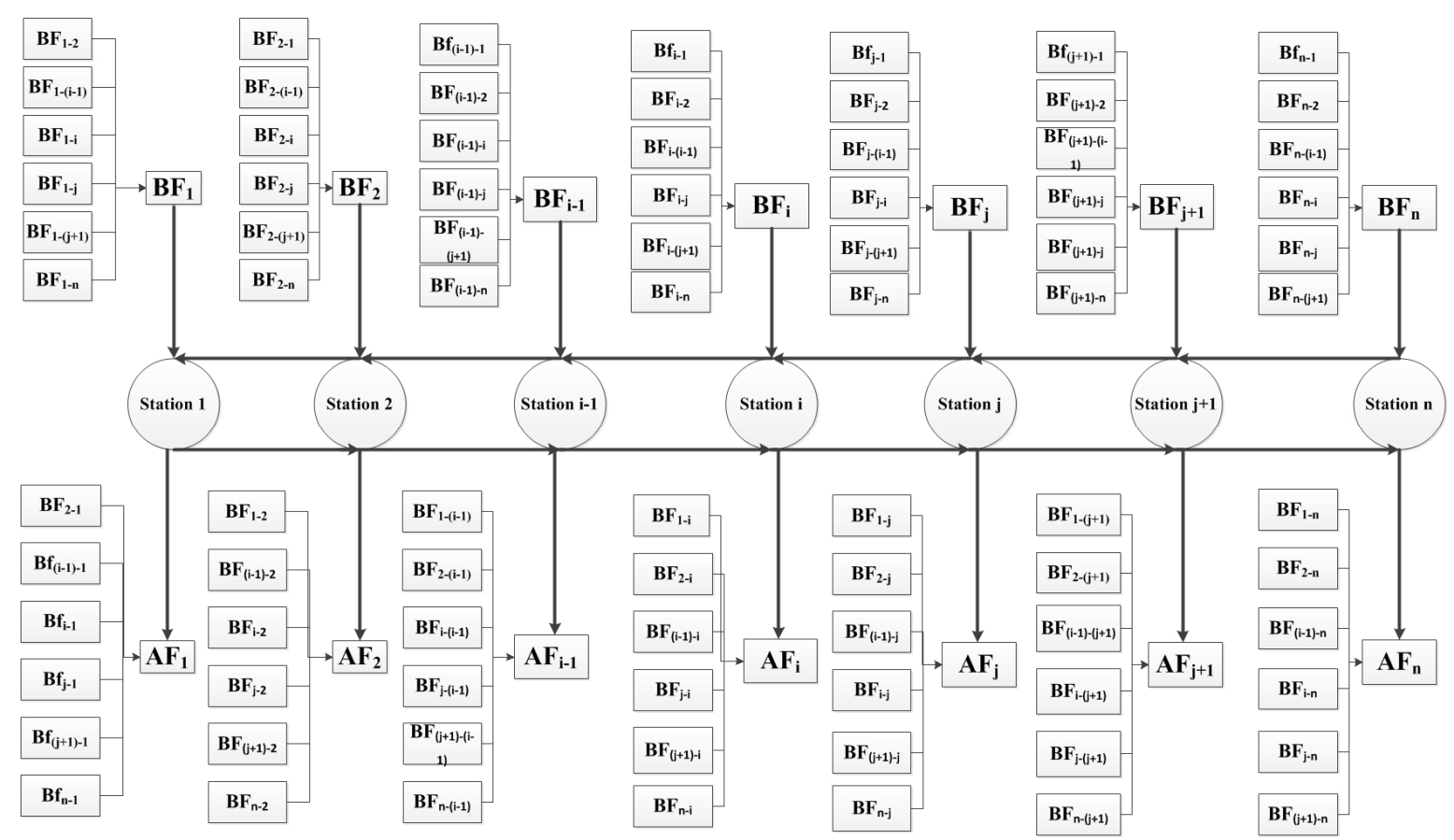

Figure 2. Composition of passenger boarding flow in individual stations. AF: alighting flow; BF: boarding flow.

As shown in Figure 1, there are two directions for a metro line: one direction starts at station 1 and terminates at station $n$; and another direction starts at station $n$ and terminates at station 1 . For measuring the crowdedness inside the vehicle between adjacent stations, only the flows in one direction need to be examined. Therefore, in referring to Figure 2, the total number of the passenger flows between the two stations $i$ and $j, \mathbf{r}_{p(i-j)}$, is composed as:

$$
\begin{gathered}
\mathrm{r}_{\mathrm{p}(i-j)}=\mathrm{BF}_{1-j}+\mathrm{BF}_{1-(j+1)}+\ldots+\mathrm{BF}_{i-n}+\mathrm{BF}_{2-j}+\mathrm{BF}_{2-(j+1)}+\ldots \mathrm{BF}_{2-n}+\ldots \mathrm{BF}_{(i-1)-j}+ \\
\mathrm{BF}_{(i-1)-(j+1)}+\ldots+\mathrm{BF}_{(i-1)-n}+\mathrm{BF}_{i-j}+\mathrm{BF}_{i-(j+1)}+\ldots \mathrm{BF}_{i-n}
\end{gathered}
$$

By applying models (3), (4), and (5) to model (2), the final measurement, model (2), can be summarized as:

$\theta_{i-j}=\frac{R \mathrm{p}_{(i-j)}}{R d_{(i-j)}}=\frac{\begin{array}{c}\mathrm{BF}_{1-j}+\mathrm{BF}_{1-(j+1)}+\ldots+\mathrm{BF}_{i-n}+\mathrm{BF}_{2-j}+\mathrm{BF}_{2-(j+1)}+\ldots \mathrm{BF}_{2-n}+\ldots \mathrm{BF}_{(i-1)-j}+ \\ \mathrm{BF}_{(i-1)-(j+1)}+\ldots+\mathrm{BF}_{(i-1)-n}+\mathrm{BF}_{i-j}+\mathrm{BF}_{i-(j+1)}+\ldots \mathrm{BF}_{i-n}\end{array}}{(x+y) z m}$

\subsection{Development of the Thresholds of Crowdedness Level}

When the data for all of the concerned parameters in model (6) are available, the crowdedness measurement $\theta_{s-s}$ can be produced. However, when the produced value of $\theta_{s-s}$ is interpreted to the level of crowdedness, there is a need to establish a threshold of crowdedness level. The study by $\mathrm{Li}$ and Hensher [38] provides a typical reference for establishing the thresholds. In their study, six levels of crowdedness for the rail line are identified, as shown in Table 1.

In Table 1, the ratio (or load factor) of passengers to seats is used to establish thresholds of crowdedness. By referring to this, the ratio (or the load factor) of passengers to the total designed 
number for both stand number and seat number is used to establish the thresholds of crowdedness in this study. Accordingly, four thresholds of $\theta_{s-s}$ are established as follows:

$$
0<\theta_{s-s} \leq \frac{x}{(x+y)}
$$

Under this situation, all of the passengers can have a seat in the metro vehicle, and no passengers need to stand; as a result, all of the passengers can receive a high-quality service from the metro system. In other words, if the value of $\theta_{s-s}$ belongs to this interval, the two adjacent stations are considered not crowded.

$$
\frac{x}{(x+y)}<\theta_{s-s} \leq 1
$$

Under this circumstance, all of the seats inside vehicle are occupied, and some passengers need to stand up. The crowdedness phenomenon appears. However, the number of passengers in this case does not exceed the designed transport volume (the sum of seat number and stand number). Therefore, the two adjacent stations are considered as having a low level of crowdedness if the value of $\theta_{s-s}$ belongs to this interval.

$$
1<\theta_{s-s} \leq \frac{x+1.5 y}{(x+y)} .
$$

Under this situation, the number of passengers exceeds the designed transport volume. Disturbance between passengers becomes obvious. This situation is considered as having a medium level of crowdedness.

$$
\frac{x+1.5 y}{(x+y)}<\theta_{s-s}
$$

In this circumstance, the number of passengers reaches the crush load of the metro vehicle. Disturbance between passengers becomes very serious. This situation refers to a high level of crowdedness between two adjacent stations.

Table 1. Thresholds for measuring the level of crowdedness in a metro line.

\begin{tabular}{ccc}
\hline & Load Factor (Passengers/Seat) & Situation of Crowding \\
\hline A & $0-0.5$ & No passenger needs to sit next to another \\
B & $0.51-0.75$ & Passengers can choose where to sit \\
C & $0.76-1$ & All passengers can sit \\
D & $1.01-1.25$ & Comfortable standee load for design \\
E & $1.26-1.5$ & Maximum schedule load \\
F & $>1.5$ & Crush load \\
\hline
\end{tabular}

\section{Case Study}

Chongqing Metro Line 3, which started operation on 29 September 2011, is selected to illustrate the application of the $\theta_{s-s}$ model. As shown in Figure 3, Line 3 in the Chongqing metro system, in blue, includes 39 stations across two big rivers (Yangtze River and Jialing River) and five districts of Chongqing. The total length of the line is $55.5 \mathrm{~km}$, which makes it the longest in the world, according to Wikipedia [39]. The line is also the busiest metro line in the whole Chongqing metro system [39]. The research team received support and assistance from staff members in the Chongqing Metro Corporation, and thus had the ability to collect relevant data about the operation of this line. As a result, the data are effective and sufficient for supporting analysis. 


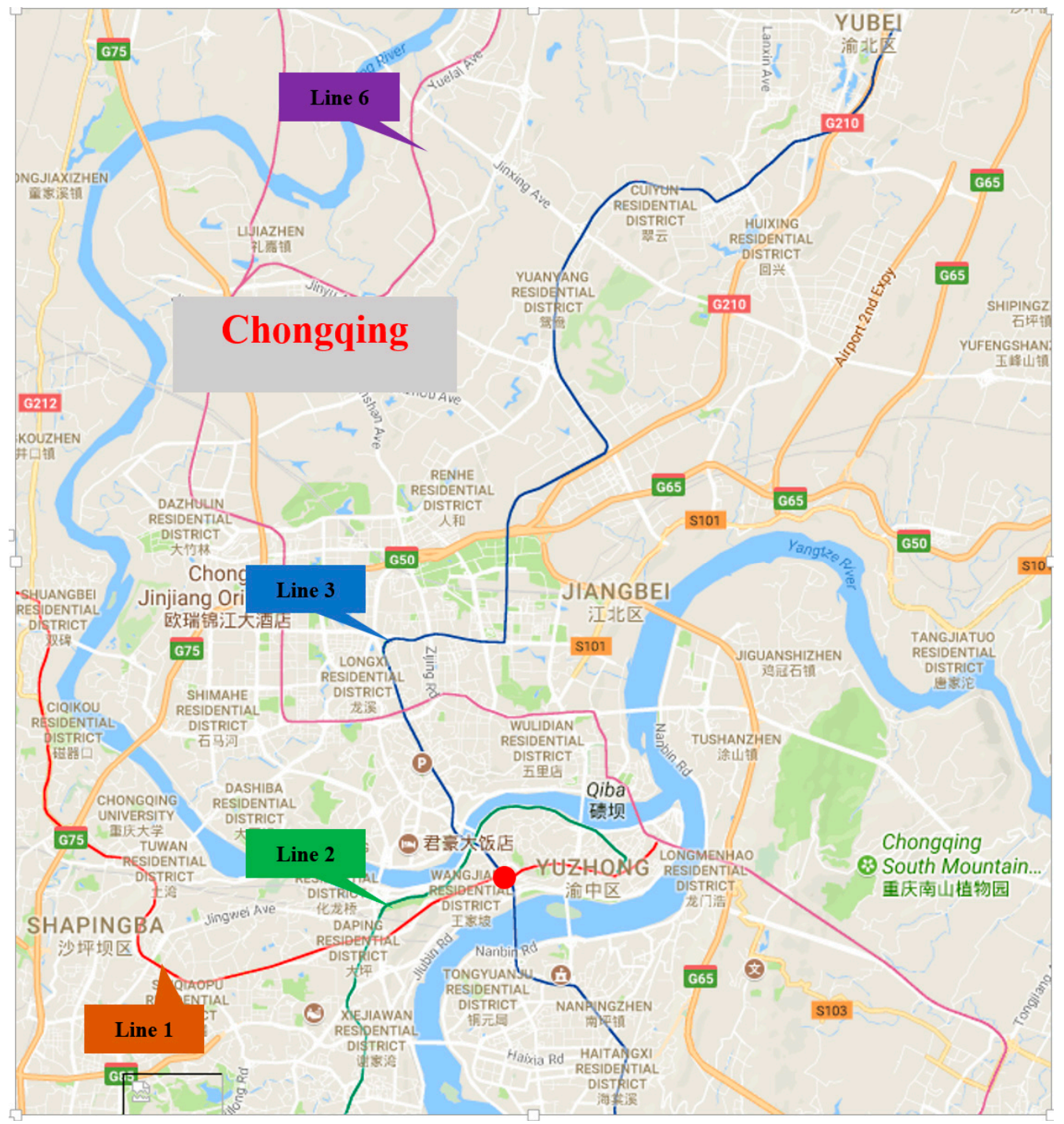

Figure 3. Chongqing metro system.

\subsection{Data Collection}

Table 2 presents the details of the 39 stations in Chongqing Metro Line 3. The flow direction from station 1 to station 39 is considered for analysis.

Table 2. Details of the stations in Chongqing Metro Line 3.

\begin{tabular}{cccccc}
\hline Station Number & Station Name & Station Number & Station Name & Station Number & Station Name \\
\hline 1 & Yudong & 14 & Sigongli & 27 & Chongqingbei Railway Station \\
2 & Jinzhu & 15 & Nanping & 28 & Longtousi \\
3 & Yuhulu & 16 & Gongmao & 29 & Tongjiayuanzi \\
4 & Xuetangwan & 17 & Tongyuanju & 30 & Jinyu \\
5 & Dashancun & 18 & Lianglukou & 31 & Jintonglu \\
6 & Huaxi & 19 & Niujiaotuo & 32 & Yuanyang \\
7 & Chalukou & 20 & Huaxinjie & 33 & The EXPO Garden \\
8 & Jiugongli & 21 & Guanyinqiao & 34 & Cuiyun \\
9 & Qilong & 22 & Hongqihegou & 35 & Changfulu \\
10 & Bagongli & 23 & Jiazhoulu & 36 & huixing \\
11 & Ertang & 24 & Zhengjiayuanzi & 37 & Shuanglong \\
12 & Liugongli & 25 & Tangjiayuanzi & 38 & Bijin \\
13 & Wugongli & 26 & Shiziping & 39 & Jiangbei Airport \\
\hline
\end{tabular}


The boarding flows (BF) for all 39 metro stations are collected for the period from 1 January 2015 to 13 June 2015. The average values of BF for all 39 stations from Monday to Sunday during the surveyed period are shown in Figure 4.

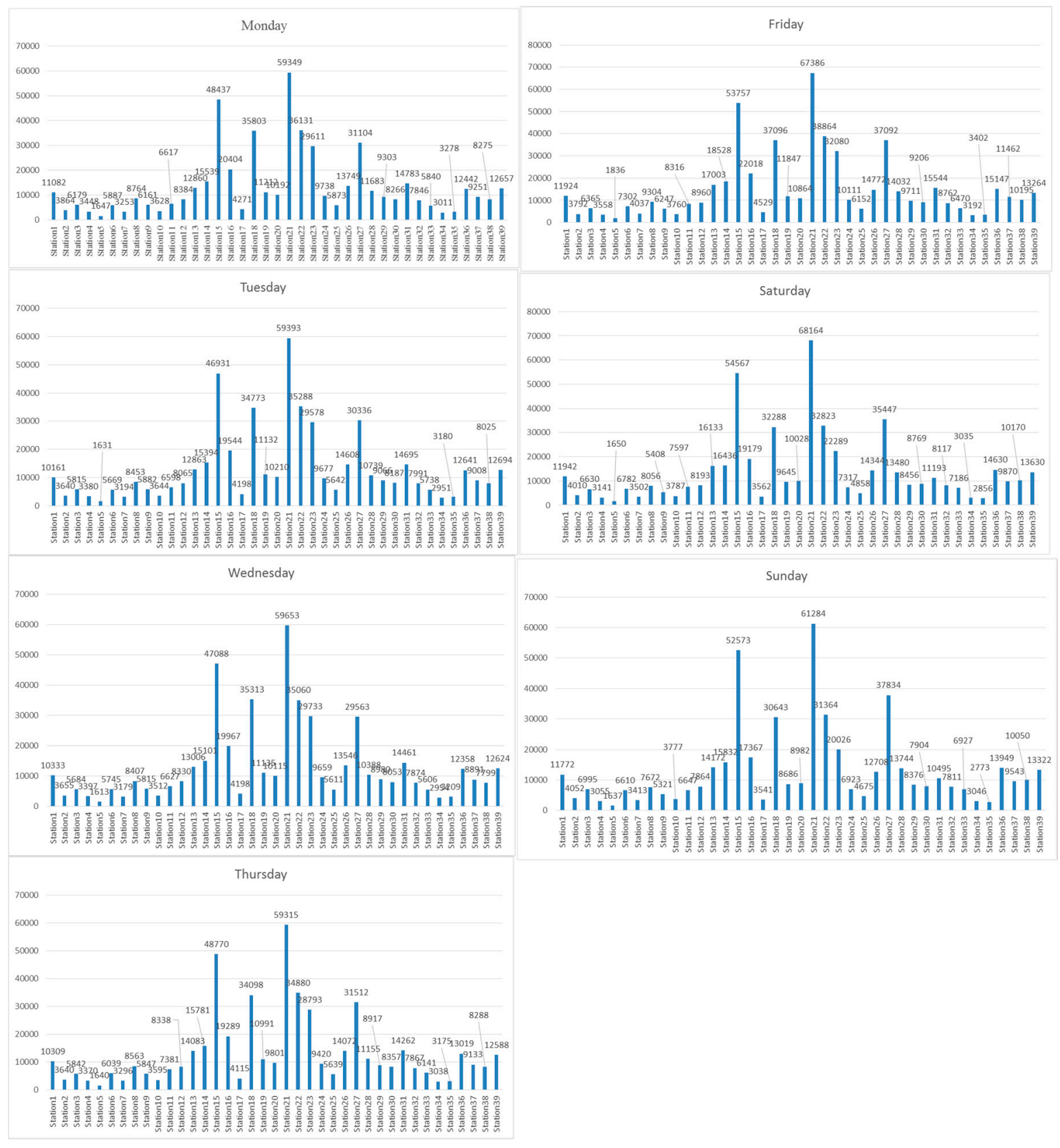

Figure 4. The value of BF for 39 metro stations.

The compositions of $\mathrm{BF}_{i}$ for each of the 39 stations are also obtained in this study. Taking $\mathrm{BF}_{1}$ as an example, Table 3 shows the compositions of $\mathrm{BF}_{1}$ for station 1 . The data of $\mathrm{BF}_{i}$ for other stations are not included in the contexts of the paper due to the limitation of the paper length. 
Table 3. Composition of passenger boarding flow for station 1. Unit: passenger.

\begin{tabular}{|c|c|c|c|c|c|c|c|}
\hline & Monday & Tuesday & Wednesday & Thursday & Friday & Saturday & Sunday \\
\hline $\mathrm{BF}_{1-2}$ & 82 & 75 & 78 & 76 & 88 & 93 & 88 \\
\hline $\mathrm{BF}_{1-3}$ & 136 & 123 & 130 & 133 & 158 & 175 & 159 \\
\hline $\mathrm{BF}_{1-4}$ & 74 & 68 & 69 & 68 & 75 & 72 & 70 \\
\hline $\mathrm{BF}_{1-5}$ & 26 & 24 & 24 & 24 & 27 & 28 & 27 \\
\hline $\mathrm{BF}_{1-6}$ & 115 & 101 & 105 & 107 & 126 & 134 & 141 \\
\hline $\mathrm{BF}_{1-7}$ & 70 & 65 & 66 & 66 & 76 & 83 & 90 \\
\hline $\mathrm{BF}_{1-8}$ & 108 & 99 & 101 & 102 & 120 & 138 & 139 \\
\hline $\mathrm{BF}_{1-9}$ & 144 & 130 & 134 & 131 & 149 & 142 & 143 \\
\hline $\mathrm{BF}_{1-10}$ & 80 & 76 & 74 & 74 & 80 & 85 & 88 \\
\hline $\mathrm{BF}_{1-11}$ & 120 & 112 & 115 & 123 & 138 & 155 & 154 \\
\hline $\mathrm{BF}_{1-12}$ & 149 & 136 & 147 & 143 & 160 & 167 & 164 \\
\hline $\mathrm{BF}_{1-13}$ & 256 & 236 & 245 & 256 & 316 & 342 & 335 \\
\hline $\mathrm{BF}_{1-14}$ & 280 & 251 & 253 & 261 & 304 & 314 & 328 \\
\hline $\mathrm{BF}_{1-15}$ & 1087 & 997 & 1022 & 1049 & 1256 & 1359 & 1262 \\
\hline $\mathrm{BF}_{1-16}$ & 475 & 429 & 447 & 423 & 508 & 468 & 426 \\
\hline $\mathrm{BF}_{1-17}$ & 73 & 70 & 71 & 70 & 80 & 78 & 77 \\
\hline $\mathrm{BF}_{1-18}$ & 939 & 875 & 887 & 859 & 964 & 825 & 811 \\
\hline $\mathrm{BF}_{1-19}$ & 267 & 249 & 251 & 244 & 265 & 209 & 200 \\
\hline $\mathrm{BF}_{1-20}$ & 183 & 171 & 174 & 167 & 191 & 196 & 182 \\
\hline $\mathrm{BF}_{1-21}$ & 1469 & 1359 & 1393 & 1355 & 1603 & 1779 & 1656 \\
\hline $\mathrm{BF}_{1-22}$ & 777 & 704 & 719 & 715 & 831 & 732 & 721 \\
\hline $\mathrm{BF}_{1-23}$ & 505 & 467 & 474 & 457 & 504 & 391 & 379 \\
\hline $\mathrm{BF}_{1-24}$ & 185 & 171 & 176 & 169 & 188 & 154 & 147 \\
\hline $\mathrm{BF}_{1-25}$ & 110 & 101 & 103 & 100 & 112 & 96 & 94 \\
\hline $\mathrm{BF}_{1-26}$ & 286 & 288 & 270 & 274 & 305 & 314 & 279 \\
\hline $\mathrm{BF}_{1-27}$ & 677 & 595 & 596 & 640 & 761 & 788 & 854 \\
\hline $\mathrm{BF}_{1-28}$ & 295 & 242 & 247 & 270 & 355 & 353 & 387 \\
\hline $\mathrm{BF}_{1-29}$ & 184 & 171 & 173 & 170 & 190 & 187 & 196 \\
\hline $\mathrm{BF}_{1-30}$ & 183 & 167 & 167 & 167 & 187 & 192 & 184 \\
\hline $\mathrm{BF}_{1-31}$ & 306 & 284 & 283 & 276 & 304 & 248 & 245 \\
\hline $\mathrm{BF}_{1-32}$ & 185 & 175 & 178 & 177 & 198 & 207 & 210 \\
\hline $\mathrm{BF}_{1-33}$ & 105 & 96 & 95 & 102 & 111 & 144 & 146 \\
\hline $\mathrm{BF}_{1-34}$ & 60 & 57 & 57 & 57 & 62 & 62 & 64 \\
\hline $\mathrm{BF}_{1-35}$ & 85 & 73 & 73 & 69 & 76 & 65 & 66 \\
\hline $\mathrm{BF}_{1-36}$ & 299 & 283 & 286 & 294 & 341 & 382 & 399 \\
\hline $\mathrm{BF}_{1-37}$ & 202 & 181 & 184 & 184 & 211 & 219 & 254 \\
\hline $\mathrm{BF}_{1-38}$ & 191 & 169 & 168 & 170 & 199 & 227 & 258 \\
\hline $\mathrm{BF}_{1-39}$ & 318 & 289 & 299 & 287 & 308 & 342 & 350 \\
\hline
\end{tabular}

\subsection{Calculation Results}

By applying the data of $\mathrm{BF}_{i-j}$ for all 39 stations to model (5), the results of the passenger flows between two adjacent stations represented by $i$ and $j$, namely $r_{p i-j}$, are calculated. The data for the other variables in model (6), including $x, y, m$, and $z$, were provided by the Chongqing Metro Corporation, with the following details:

$$
x=48 ; y=100 ; \mathrm{m}=180 ; z=6
$$

By applying these variable values and the value of $r_{p(i-j)}$ to model (6), the values of parameter $\theta_{i-j}$ are produced, as shown in Table 4 . Based on the calculation results for the variable $\theta_{i-j}$, it can be found that the crowdedness between two adjacent stations along Chongqing Metro Line 3 varies significantly. For further discussion on the crowdedness level between different pairs of adjacent stations on this metro line, the thresholds for each crowdedness level need to be established. By referring to the four threshold scenarios discussed in the previous section, the following four thresholds of crowdedness level are established for the case concerned in this study:

(a) when $0<\theta_{i-j} \leq 0.32$, it indicates a situation of no crowdedness.

(b) when $0.32<\theta_{i-j} \leq 1$, it indicates a low level of crowdedness.

(c) when $1<\theta_{i-j} \leq 1.1$, it indicates a medium level of crowdedness.

(d) when $1.1<\theta_{i-j}$, it indicates a high level of crowdedness. 
Table 4. The value of $\theta_{i-j}$ and level of crowdedness between adjacent stations on Chongqing Metro Line 3.

\begin{tabular}{|c|c|c|c|c|c|c|c|c|c|c|c|c|c|c|}
\hline & \multicolumn{2}{|c|}{ Monday } & \multicolumn{2}{|c|}{ Tuesday } & \multicolumn{2}{|c|}{ Wednesday } & \multicolumn{2}{|c|}{ Thursday } & \multicolumn{2}{|r|}{ Friday } & \multicolumn{2}{|c|}{ Saturday } & \multicolumn{2}{|c|}{ Sunday } \\
\hline & $\theta_{i-j}$ & $\begin{array}{l}\text { Crowdedness } \\
\text { Results }\end{array}$ & $\theta_{i-j}$ & $\begin{array}{l}\text { Crowdedness } \\
\text { Results }\end{array}$ & $\theta_{i-j}$ & $\begin{array}{c}\text { Crowdedness } \\
\text { Results }\end{array}$ & $\theta_{i-j}$ & $\begin{array}{c}\text { Crowdedness } \\
\text { Results }\end{array}$ & $\theta_{i-j}$ & $\begin{array}{l}\text { Crowdedness } \\
\text { Results }\end{array}$ & $\theta_{i-j}$ & $\begin{array}{c}\text { Crowdedness } \\
\text { Results }\end{array}$ & $\theta_{i-j}$ & $\begin{array}{c}\text { Crowdedness } \\
\text { Results }\end{array}$ \\
\hline station1-2 & 0.08 & Not & 0.08 & Not & 0.08 & Not & 0.08 & Not & 0.09 & Not & 0.09 & Not & 0.09 & Not \\
\hline station2-3 & 0.11 & Not & 0.1 & Not & 0.1 & Not & 0.1 & Not & 0.12 & Not & 0.12 & Not & 0.12 & Not \\
\hline station3-4 & 0.15 & Not & 0.14 & Not & 0.14 & Not & 0.14 & Not & 0.16 & Not & 0.16 & Not & 0.17 & Not \\
\hline station4-5 & 0.18 & Not & 0.17 & Not & 0.17 & Not & 0.17 & Not & 0.18 & Not & 0.19 & Not & 0.19 & Not \\
\hline station5-6 & 0.19 & Not & 0.18 & Not & 0.18 & Not & 0.18 & Not & 0.2 & Not & 0.2 & Not & 0.2 & Not \\
\hline station6-7 & 0.23 & Not & 0.22 & Not & 0.22 & Not & 0.22 & Not & 0.25 & Not & 0.24 & Not & 0.24 & Not \\
\hline station7-8 & 0.25 & Not & 0.24 & Not & 0.24 & Not & 0.24 & Not & 0.27 & Not & 0.26 & Not & 0.27 & Not \\
\hline station8-9 & 0.31 & Not & 0.29 & Not & 0.29 & Not & 0.3 & Not & 0.34 & Low & 0.32 & Not & 0.32 & Not \\
\hline station9-10 & 0.35 & Low & 0.33 & Low & 0.33 & Low & 0.33 & Low & 0.37 & Low & 0.35 & Low & 0.35 & Low \\
\hline station10-11 & 0.37 & Low & 0.35 & Low & 0.35 & Low & 0.36 & Low & 0.4 & Low & 0.37 & Low & 0.38 & Low \\
\hline station11-12 & 0.41 & Low & 0.39 & Low & 0.39 & Low & 0.4 & Low & 0.45 & Low & 0.42 & Low & 0.41 & Low \\
\hline station12-13 & 0.46 & Low & 0.44 & Low & 0.44 & Low & 0.45 & Low & 0.5 & Low & 0.47 & Low & 0.46 & Low \\
\hline station13-14 & 0.55 & Low & 0.51 & Low & 0.51 & Low & 0.53 & Low & 0.6 & Low & 0.56 & Low & 0.55 & Low \\
\hline station14-15 & 0.71 & Low & 0.67 & Low & 0.67 & Low & 0.69 & Low & 0.77 & Low & 0.72 & Low & 0.71 & Low \\
\hline station15-16 & 0.98 & Low & 0.96 & Low & 0.98 & Low & 0.99 & Low & 1 & Low & 0.96 & Low & 0.94 & Low \\
\hline station16-17 & 1.04 & Medium & 1.02 & Medium & 1.04 & Medium & 1.03 & Medium & 1.07 & Medium & 1.01 & Medium & 1.03 & Medium \\
\hline station17-18 & 1.06 & Medium & 1.04 & Medium & 1.05 & Medium & 1.04 & Medium & 1.08 & Medium & 1.02 & Medium & 1.05 & Medium \\
\hline station18-19 & 1.17 & High & 1.15 & High & 1.12 & High & 1.15 & High & 1.19 & High & 1.13 & High & 1.08 & Medium \\
\hline station19-20 & 1.2 & High & 1.18 & High & 1.13 & High & 1.16 & High & 1.21 & High & 1.14 & High & 1.11 & High \\
\hline station20-21 & 1.22 & High & 1.2 & High & 1.14 & High & 1.18 & High & 1.22 & High & 1.16 & High & 1.14 & High \\
\hline station21-22 & 1.26 & High & 1.27 & High & 1.16 & High & 1.22 & High & 1.3 & High & 1.21 & High & 1.18 & High \\
\hline station22-23 & 1.23 & High & 1.24 & High & 1.15 & High & 1.21 & High & 1.31 & High & 1.21 & High & 1.14 & High \\
\hline station23-24 & 1.15 & High & 1.16 & High & 1.11 & High & 1.17 & High & 1.25 & High & 1.18 & High & 1.17 & High \\
\hline station24-25 & 1.13 & High & 1.13 & High & 1.12 & High & 1.15 & High & 1.23 & High & 1.16 & High & 1.18 & High \\
\hline station25-26 & 1.11 & High & 1.12 & High & 1.1 & High & 1.14 & High & 1.21 & High & 1.15 & High & 1.17 & High \\
\hline station26-27 & 1.04 & Medium & 1.08 & Medium & 1.05 & Medium & 1.03 & Medium & 1.09 & Medium & 1.1 & High & 1.14 & High \\
\hline station27-28 & 0.82 & Low & 0.81 & Low & 0.75 & Low & 0.73 & Low & 0.79 & Low & 0.79 & Low & 0.83 & Low \\
\hline station28-29 & 0.73 & Low & 0.69 & Low & 0.69 & Low & 0.66 & Low & 0.7 & Low & 0.72 & Low & 0.73 & Low \\
\hline station29-30 & 0.68 & Low & 0.65 & Low & 0.65 & Low & 0.62 & Low & 0.66 & Low & 0.67 & Low & 0.69 & Low \\
\hline station30-31 & 0.63 & Low & 0.61 & Low & 0.61 & Low & 0.58 & Low & 0.61 & Low & 0.63 & Low & 0.65 & Low \\
\hline station31-32 & 0.54 & Low & 0.53 & Low & 0.53 & Low & 0.5 & Low & 0.51 & Low & 0.53 & Low & 0.55 & Low \\
\hline station32-33 & 0.48 & Low & 0.47 & Low & 0.47 & Low & 0.45 & Low & 0.45 & Low & 0.48 & Low & 0.48 & Low \\
\hline station33-34 & 0.45 & Low & 0.45 & Low & 0.45 & Low & 0.41 & Low & 0.42 & Low & 0.44 & Low & 0.44 & Low \\
\hline station34-35 & 0.44 & Low & 0.43 & Low & 0.43 & Low & 0.4 & Low & 0.4 & Low & 0.42 & Low & 0.42 & Low \\
\hline station35-36 & 0.41 & Low & 0.4 & Low & 0.4 & Low & 0.37 & Low & 0.38 & Low & 0.4 & Low & 0.39 & Low \\
\hline station36-37 & 0.24 & Not & 0.23 & Not & 0.23 & Not & 0.23 & Not & 0.25 & Not & 0.28 & Not & 0.28 & Not \\
\hline station37-38 & 0.18 & Not & 0.17 & Not & 0.17 & Not & 0.17 & Not & 0.18 & Not & 0.19 & Not & 0.19 & Not \\
\hline station38-39 & 0.11 & Not & 0.11 & Not & 0.11 & Not & 0.11 & Not & 0.11 & Not & 0.11 & Not & 0.11 & Not \\
\hline
\end{tabular}


By referring the four above-mentioned thresholds to the value of $\theta_{i-j}$ in Table 4 , the crowdedness levels among 38 pairs of adjacent stations on Chongqing Metro Line 3 are also identified and summarized in Table 4.

\subsection{Discussion}

The calculated results in Table 4 suggest that those stations at the ends of the metro line are usually not crowded, typically from station 1 to station 9 , and from station 36 to station 39 . The produced value of the crowdedness variable $\theta_{i-j}$ between these stations are all lower than 0.32 . This indicates that passengers can normally have seats when traveling to these stations, and will not feel crowded. When passengers travel from station 9 to station 16, and from station 27 to station 36, they may not have seats, and need to stand in the metro carriage, but will not feel crowded, as the value of $\theta_{i-j}$ for these stations are more than 0.32 and lower than 1 . In other words, the crowdedness level in each of these stations is low. Furthermore, the information in Table 4 suggests that the adjacent stations 16-17, 17-18, and 26-27 are at a medium level of crowdedness, where the value of $\theta_{i-j}$ in these stations is close to 1.1. Nevertheless, it appears that the adjacent stations from station 18 to station 26 are at a high level of crowdedness, as the value of $\theta_{i-j}$ for them is always above 1.1.

Furthermore, it has been found that the stations with a high level of crowdedness, namely, from station 18 to station 26, are located in the central area of Metro Line 3. The most crowded traveling occurs between stations 21 and 22, with the values of $\theta_{21-22}$ from Monday to Sunday equivalent to $1.26,1.27,1.16,1.22,1.30,1.21$, and 1.18, respectively. These crowdedness in adjacent stations occurs in two circumstances. In the first case, they are close to the central business district (CBD), or they are designed as a traffic hub, where many people will use the metro line [32]. For example, station 21 (Guanyingqiao) is the closest to the CBD, and station 27 (Chongqingbei) is close to the railway station. This can be echoed by the data in Figure 3, where it can be found that the average boarding flows on Sunday in these two stations are ranked in the top three among all 39 stations, with passenger numbers of 61,284 and 37,834 , respectively. The high ridership density induces the high level of crowdedness between these adjacent stations, and this high level of crowdedness remains consistent throughout the week, as shown in Table 2.

In another circumstance, it can be noted that these transfer stations are all metro stations with a high level of crowdedness. For example, as shown in Table 4, stations 18 and 19 both have a high level of crowdedness, where station 18 (Lianglukou) is the transfer station between Line 3 and Line 1, and station 19 (Niujiaotuo) is the transfer station between Line 3 and Line 2.

The above analysis results were presented to a group of staff of the Chongqing Metro Corporation who work on Metro Line 3. The discussion suggested that the calculation results are consistent with what the work staff had been experiencing in practice. The work staff pointed out that the crowdedness is severe in the stations located in the middle areas. One of the main reasons suggested for the crowdedness problem is the insufficient carrying capacity of the metro line, particularly during peak hours. The crowdedness inside the carriages results in long waiting times on the platforms of the stations, which causes severe crowdedness at the metro stations.

Considering that the urban metro system is public transportation, the government and relevant administrative departments of the metro system should play a leading role in developing appropriate policy measures and planning decisions to mitigate the crowdedness problem, especially for the stations with a high level of crowdedness. In this study, two categories of policy measures have been discussed for reducing crowdedness among metro stations and providing some reference for governmental administration. One is increasing the vehicle frequency among crowded metro stations. The Chongqing Metro Corporation adopted a sectional operation approach to increase the frequency of the metro vehicles. Through this approach, the operation for the 39 stations on Metro Line 3 is divided into three sections during peak hours: section one, starting with station 1 and ending in station 24; section two, starting with station 8 and ending in station 24; and section three, starting with station 14 and ending in station 39 . Section two, which covers more central areas, is scheduled with more vehicle 
frequency during peak hours, and can improve the carrying capacity among the crowded stations. It was reported during the discussion with the work staff that this sectional operation method does relieve the crowdedness both inside carriages and on the platforms.

The other policy measure would involve implementing a variable ticket price. According to previous research results, the ticket price is one of the main factors that determines whether or not passengers will choose the metro system as a means of transport [40-42]. Therefore, through application of dynamic ticket pricing, authorities can consider this as a potential policy that would decrease the number of metro passengers during peak hours, and thus reduce crowdedness. For example, the Australia Rail Company implemented a category of rail ticket i.e., early bird ticket, which offered passengers free rail travel if their trips were completed before 7 am in Melbourne. This ticket has reduced demand during peak time between $1.2 \%$ and $1.5 \%$ from previous levels [19]. The New York metro system in the US also implemented two categories tickets, namely, peak ticket and off-peak ticket, in a day. Passengers need to purchase peak tickets between 5-10 a.m. and 4-8 p.m. It is important to note that the fare of the two ticket categories are totally different, and the off-peak fare is only a quarter of the peak fare [43]. At present, dynamic ticket pricing is not used by the Chongqing Metro Corporation. Therefore, in the future, according to the experience of cities such as Melbourne, New York, and others, the Chongqing Metro Corporation may put forward the policy for different metro ticket pricing in Chongqing to reduce the crowdedness problem.

\section{Conclusions}

Crowdedness in operating metro systems has presented a major problem in many cities, and therefore, methods are needed to mitigate the problem. In order to adopt effective methods, there is a need for an effective tool for measuring the level of crowdedness. Through introducing a measurement model, this study suggested that the level of crowdedness between adjacent stations in a metro line can be effectively measured. The results from the analysis can inform where the most crowded stations are in a metro system, and then adequate management strategies and methods can be identified to reduce the problem. The effectiveness of the proposed model is demonstrated from the case study of Chongqing Metro Line 3. From the case study, it has been found that the most crowded linkages between the metro stations are either in the central area of the metro line or the stations for transferring between different lines. More attention should be given to these stations in order to reduce the risks of accidents. The development of the model in this paper adds value to the development of methodology for further studies on the utility performance of metro systems.

The limitation of this paper is that the numbers for boarding flow and alighting flow may not be uniform if passengers are boarding from other lines through the transfer stations. The lack of this consideration may affect the adequacy of evaluation results if the evaluation is requested from a holistic point of view. It is appreciated that the case study used the daily total passenger flow data of each station. However, the distribution of the passenger flow throughout a day is also an important and interesting factor to be considered, especially for peak hours and off-peak hours. As a result, this issue is on the future research agenda for our research team.

Acknowledgments: This research is funded by the Chongqing Federation of Social Science (Project No: 2017QNGL52).

Author Contributions: Liudan Jiao designed the research and drafted the manuscript. Liyin Shen and Chenyang Shuai analyzed data and provided extensive revisions on the manuscript. Yongtao Tan and Bei He analyzed data and drafted the manuscript.

Conflicts of Interest: The authors declare no conflict of interest.

\section{References}

1. Loo, B.P.; Cheng, A.H. Are there useful yardsticks of population size and income level for building metro systems? Some worldwide evidence. Cities 2010, 27, 299-306. [CrossRef] 
2. Andrade, C.E.S.; D'Agosto, M.A. The Role of Rail Transit Systems in Reducing Energy and Carbon Dioxide Emissions: The Case of the City of Rio de Janeiro. Sustainability 2016, 8, 150. [CrossRef]

3. Sun, D.J.; Zhao, Y.; Lu, Q.C. Vulnerability analysis of urban rail transit networks: A case study of Shanghai, China. Sustainability 2015, 7, 6919-6936. [CrossRef]

4. Shen, L.Y.; Jiao, L.D.; Zhou, J.Y.; Ren, W.J. Evaluation indicators for the efficiency of metro systems from a socio-economic-technical perspective. In Proceedings of the 18th International Symposium on Advancement of Construction Management and Real Estate, Xi'an, China, 22 May 2014.

5. Chen, F.; Shen, X.; Wang, Z.; Yang, Y. An Evaluation of the Low-Carbon Effects of Urban Rail Based on Mode Shifts. Sustainability 2017, 9, 401. [CrossRef]

6. Zhang, X.; Liu, X.; Hang, J.; Yao, D.; Shi, G. Do Urban Rail Transit Facilities Affect Housing Prices? Evidence from China. Sustainability 2016, 8, 380. [CrossRef]

7. Litman, T. Evaluating rail transit benefits: A comment. Transp. Policy 2007, 14, 94-97. [CrossRef]

8. Metrobits. Available online: http:// mic-ro.com/metro/ (accessed on 22 May 2015).

9. Observer. Available online: http://www.guancha.cn/Project/2013_03_14_131915.shtml (accessed on 14 March 2013).

10. Shen, L.Y.; Jiao, L.D.; He, B.; Li, L.C. Evaluation on the utility efficiency of metro infrastructure projects in China from sustainable development perspective. Int. J. Proj. Manag. 2015, 33, 528-536. [CrossRef]

11. Jiang, S.; Sun, Y.; Du, Y. Influence of in-vehicle congestion degree on choice of public transit mode. J. Tongji Univ. Nat. Sci. 2012, 40, 1831-1835.

12. Tirachini, A.; Hensher, D.A.; Rose, J.M. Crowding in public transport systems: Effects on users, operation and implications for the estimation of demand. Transp. Res. Part A Policy Pract. 2013, 53, 36-52. [CrossRef]

13. Cox, T.; Houdmont, J.; Griffiths, A. Rail passenger crowding, stress, health and safety in Britain. Transp. Res. Part A Policy Pract. 2006, 40, 244-258. [CrossRef]

14. Kim, H.; Kwon, S.; Wu, S.K.; Sohn, K. Why do passengers choose a specific car of a metro train during the morning peak hours? Transp. Res. Part A Policy Pract. 2014, 61, 249-258. [CrossRef]

15. Li, Z.; Hensher, D.A. Crowding and public transport: A review of willingness to pay evidence and its relevance in project appraisal. Transp. Policy 2011, 18, 880-887. [CrossRef]

16. Raveau, S.; Guo, Z.; Muñoz, J.C.; Wilson, N.H. A behavioural comparison of route choice on metro networks: Time, transfers, crowding, topology and socio-demographics. Transp. Res. Part A Policy Pract. 2014, 66, 185-195. [CrossRef]

17. Kim, K.M.; Hong, S.P.; Ko, S.J.; Kim, D. Does crowding affect the path choice of metro passengers? Transp. Res. Part A Policy Pract. 2015, 77, 292-304. [CrossRef]

18. Kuby, M.; Barranda, A.; Upchurch, C. Factors influencing light-rail station boardings in the United States. Transp. Res. Part A Policy Pract. 2004, 38, 223-247. [CrossRef]

19. Currie, G. Quick and effective solution to rail overcrowding: Free early bird ticket experience in Melbourne, Australia. Transp. Res. Rec. J. Transp. Res. Board 2010, 2146, 35-42. [CrossRef]

20. Wardman, M.; Whelan, G. Twenty years of rail crowding valuation studies: Evidence and lessons from British experience. Transp. Rev. 2011, 31, 379-398. [CrossRef]

21. Evans, G.W.; Wener, R.E. Crowding and personal space invasion on the train: Please don't make me sit in the middle. J. Environ. Psychol. 2007, 27, 90-94. [CrossRef]

22. Yook, D.; Heaslip, K. The effect of crowding on public transit user travel behavior in a large-scale public transportation system through modeling daily variations. Transp. Plan. Technol. 2015, 38, 935-953. [CrossRef]

23. Mahudin, N.D.M.; Cox, T.; Griffiths, A. Measuring rail passenger crowding: Scale development and psychometric properties. Transp. Res. Part F Traffic Psychol. Behav. 2012, 15, 38-51. [CrossRef]

24. Wu, J.; Ma, S. Crowdedness classification method for island platform in metro station. J. Transp. Eng. 2012, 139, 612-662. [CrossRef]

25. Chow, W.K. 'Waiting time' for evacuation in crowded areas. Build. Environ. 2007, 42, 3757-3761. [CrossRef]

26. Tirachini, A.; Sun, L.; Erath, A.; Chakirov, A. Valuation of sitting and standing in metro trains using revealed preferences. Transp. Policy 2016, 47, 94-104. [CrossRef]

27. Qin, F. Investigating the in-vehicle crowding cost functions for public transit modes. Math. Prob. Eng. 2014, 2014, 502708. [CrossRef]

28. Whelan, G.; Crockett, J. An investigation of the willingness to pay to reduce rail overcrowding. In Proceedings of the First International Conference on Choice Modelling, Harrogate, UK, 24 March 2009. 
29. Li, M.; Wang, Y.H.; Jia, L.M. Passenger flow modes and control strategies in urban rail transit station. J. Southeast Univ. 2015, 1203-1208. [CrossRef]

30. Zhang, L.Y.; Meng, L.; Yin, Q. Classification of Urban Rail Transit Stations based on SAX. J. GEO-Inf. Sci. 2016, 18, 1597-1607.

31. Yin, Q.; Zhang, L.Y.; Meng, L. Classification of subway stations in Beijing based on passenger flow characteristics. Prog. Geogr. 2016, 35, 126-134.

32. Zhao, J.; Deng, W.; Song, Y.; Zhu, Y. What influences Metro station ridership in China? Insights from Nanjing. Cities 2013, 35, 114-124. [CrossRef]

33. Li, L.; Ren, H.; Zhao, S.; Duan, Z.; Zhang, Y.; Zhang, A. Two dimensional accessibility analysis of metro stations in Xi'an, China. Transp. Res. Part A Policy Pract. 2017, 106, 414-426. [CrossRef]

34. Wei, J.L.; Liang, P.; Yan, Y.; Jiao, Y. A Study of Optimization of Station Distribution in Urban Rail Transit. J. Qingdao Technol. Univ. 2008, 29, 88-93.

35. Wikipedia. 2015a. Available online: https:/ / en.wikipedia.org/wiki/Crowd (accessed on 26 June 2015).

36. Wikipedia. 2015b. Available online: https:/ / en.wikipedia.org/wiki/Crowd_counting (accessed on 26 June 2015).

37. Jiao, L.D.; Shuai, C.Y.; Shen, L.Y.; Luo, X.C. The level of crowdedness in operating metro systems in the selected cities in China. In Proceedings of the 20th International Symposium on Advancement of Construction Management and Real Estate, Hangzhou, China, 23-25 October 2016.

38. Li, Z.; Hensher, D.A. Crowding in public transport: A review of objective and subjective measures. J. Public Transp. 2013, 16, 107-134. [CrossRef]

39. Wikipedia. 2015c. Available online: https://en.wikipedia.org/wiki/Line_3,_Chongqing_Rail_Transit (accessed on 26 June 2015).

40. Zhou, X.; Zhang, X.; Yang, X.; Lao, Y. Travel mode choice-based prediction of public transit demand. J.-Tongji Univ. 2007, 35, 1627-1631.

41. Zhu, Y.; Wang, Y.; Ding, C. Investigating the influential factors in the metro choice behavior: Evidences from Beijing, China. KSCE J. Civ. Eng. 2016, 20, 2947-2954. [CrossRef]

42. Cheng, Y.H.; Tseng, W.C. Exploring the effects of perceived values, free bus transfer, and penalties on intermodal metro-bus transfer users' intention. Transp. Policy 2016, 47, 127-138. [CrossRef]

43. Qiao, Y.F. Research on Differential Pricing Issue of China's Metro Tickets. Prices Mon. 2015, 2, 42-45.

(C) 2017 by the authors. Licensee MDPI, Basel, Switzerland. This article is an open access article distributed under the terms and conditions of the Creative Commons Attribution (CC BY) license (http:/ / creativecommons.org/licenses/by/4.0/). 